\title{
Paley-Wiener-Type Theorem for a Class of Integral Transforms Arising from a Singular Dirac System
}

\author{
A. I. Zayed and V. K. Tuan
}

\begin{abstract}
A characterization of weighted $L_{2}(I)$ spaces in terms of their images under various integral transformations is derived, where $I$ is a finite interval. The class of integral transformations considered is related to certain singular Dirac systems on a half line.
\end{abstract}

Keywords: Paley-Wiener theorem, singular Dirac system

AMS subject classification: Primary 44 A 15, 34 B 24, secondary 42 B 10, 33 C 45

\section{Introduction}

The Paley-Wiener theorem [5] gives a characterization of the space $L_{2}[-\sigma, \sigma]$ in terms of its image under the Fourier transformation by showing that a function $f(x)$ is in $L_{2}[-\sigma, \sigma]$ if and only if its Fourier transform $\hat{f}(\omega)$ can be continued analytically to the whole complex plane as an entire function of exponential type at most $\sigma$ whose restriction to the real axis belongs to $L_{2}(R)$. This characterization uses complex-variable techniques and as a result it does not lend itself very naturally to other more complicated integral transformations.

Alternative approaches using real analysis techniques have been developed in the last few years to characterize the images of spaces of the form $L_{2}[I, d \rho]$, for some measure $d \rho$ and an interval $I$ (finite or infinite), under various integral transformations, such as the Mellin [11], Hankel [10], $Y$ [9], and Airy transforms [12].

One of the first and simplest of such results was discovered by H. Bang [2]. It can be rephrased as follows: let $f \in C^{\infty}(\mathbb{R})$ be such that all its derivatives belong to $L_{2}(\mathbb{R})$. Then

$$
\lim _{n \rightarrow \infty}\left\|f^{(n)}\right\|_{2}^{\frac{1}{n}}=\sup \{|y|: y \in \operatorname{supp} \hat{f}\} .
$$

In particular, a function $f$ is the Fourier transform of a square integrable function vanishing outside the interval $[-\sigma, \sigma]$ if and only if

$$
\lim _{n \rightarrow \infty}\left\|f^{(n)}\right\|_{2}^{\frac{1}{n}} \leq \sigma
$$

A. I. Zayed: Univ. Central Florida, Dept. Math., Orlando, FL 32816, USA

V. K. Tuan: Kuwait Univ., Dept. Math. \& Comp. Sci., P.O. Box 5969, Safat 13060, Kuwait zayed@pegasus.cc.ucf.edu and vu@sci.kuniv.edu.kw 
In a recent paper [13], we proposed a unified approach to handle a large class of integral transforms that includes the Fourier sine and cosine transforms, the Hankel, the Weber, the Kontorovich-Lebedev and the Jacobi transforms. This class of integral transforms arises from two types of singular Sturm-Liouville problems: singular on a half line and singular on the whole line. The main idea of this approach goes as follows:

Let $L$ be a differential operator, with a continuous spectrum $\Omega_{1}$, and $\phi(x, \lambda)$ be an eigenfunction with corresponding eigenvalue $\lambda: L \phi=-\lambda \phi$. In addition, suppose that $T: L_{2}\left(\Omega_{1} ; d \rho_{1}\right) \rightarrow L_{2}\left(\Omega_{2} ; d \rho_{2}\right)$,

$$
f(x)=(T F)(x)=\int_{\Omega_{1}} F(\lambda) \phi(x, \lambda) d \rho_{1}(\lambda)
$$

is a unitary transformation, i.e.

$$
\int_{\Omega_{2}}|f(x)|^{2} d \rho_{2}(x)=\int_{\Omega_{1}}|F(\lambda)|^{2} d \rho_{1}(\lambda) .
$$

In that case, if $\lambda^{n} F(\lambda) \in L_{2}\left(\Omega_{1} ; d \rho_{1}\right)$ for any $n \in \mathbb{N}_{0}$, we have

$$
L^{n} f(x)=\int_{\Omega_{1}}(-\lambda)^{n} F(\lambda) \phi(x, \lambda) d \rho_{1}(\lambda)
$$

and

$$
\int_{\Omega_{2}}\left|L^{n} f(x)\right|^{2} d \rho_{2}(x)=\int_{\Omega_{1}}\left|\lambda^{n} F(\lambda)\right|^{2} d \rho_{1}(\lambda) .
$$

Raising both sides of (1) to the power $\frac{1}{2 n}$ and taking the limit as $n \rightarrow \infty$ we get [8]

$$
\lim _{n \rightarrow \infty}\left\|L^{n} f\right\|_{L_{2}\left(\Omega_{2} ; d \rho_{2}\right)}^{\frac{1}{n}}=\sup _{\lambda \in \operatorname{supp} F}|\lambda|
$$

where $\operatorname{supp} F$ denotes the support of $F$, the smallest closed set, outside which the function $F$ vanishes almost everywhere. From (2) it is obvious that if

$$
\lim _{n \rightarrow \infty}\left\|L^{n} f\right\|_{L_{2}\left(\Omega_{2} ; d \rho_{2}\right)}^{\frac{1}{n}}<\infty
$$

then $F$ has a compact support. Hence, formula (2) plays a decisive role in studying integral transforms of functions with compact supports. It can be shown that under some "extra conditions" on $f$ inequality (3) gives the necessary and sufficient condition for a function $f$ to be a $T$-transform of a function $F \in L_{2}\left(\Omega_{1} ; d \rho_{1}\right)$ with compact support. Formula (3) yields Bang's formula when $\Omega_{1}=(-\infty, \infty), \phi(x, \lambda)=e^{i x \lambda}, d \rho_{1}(\lambda)=d \lambda$ and $L=\frac{d}{d x}$.

In this paper we extend this technique to study integral transforms arising from singular Dirac systems of differential equations [4]. The image of the space of functions with compact supports under those transforms is fully described. As examples, the Hartley transform and some transforms with the Airy and the Bessel functions as kernels are considered. 


\section{Singular Dirac system}

Consider the one-dimensional singular Dirac system on the half line

$$
\left.\begin{array}{rl}
y_{2}^{\prime}+p_{11}(x) y_{1}+p_{12}(x) y_{2} & =\lambda y_{1} \\
-y_{1}^{\prime}+p_{21}(x) y_{1}+p_{22}(x) y_{2} & =\lambda y_{2}
\end{array}\right\} \quad(0<x<\infty)
$$

where $p_{i j}(i, j=1,2)$ are real-valued functions and $\lambda$ is a parameter. Using matrix notation, we can write system (4) in the form

$$
B Y^{\prime}+P(x) Y=\lambda Y
$$

where

$$
B=\left(\begin{array}{cc}
0 & 1 \\
-1 & 0
\end{array}\right), \quad P(x)=\left(\begin{array}{ll}
p_{11}(x) & p_{12}(x) \\
p_{21}(x) & p_{22}(x)
\end{array}\right), \quad Y=\left(\begin{array}{l}
y_{1}(x) \\
y_{2}(x)
\end{array}\right)
$$

In the case when $p_{12}=p_{21}=0, p_{11}(x)=V(x)+m, p_{22}(x)=V(x)-m, V$ is a potential function, and $m$ is the mass of a particle, system (4) is called a one-dimensional stationary Dirac system in relativistic quantum theory.

Under the assumption $p_{12}=p_{21}$ we can transform system (4) into the canonical form

$$
B Z^{\prime}+Q(x) Z=\lambda Z
$$

where

$$
\begin{aligned}
Y & =H(x) Z \\
H(x) & =\left(\begin{array}{cc}
\cos \phi(x) & -\sin \phi(x) \\
\sin \phi(x) & \cos \phi(x)
\end{array}\right) \\
Q(x) & =H^{-1}(x) B \frac{d}{d x} H(x)+H^{-1}(x) P(x) H(x) .
\end{aligned}
$$

In addition, if we choose

$$
\phi(x)= \begin{cases}\frac{1}{2} \tan ^{-1}\left(\frac{2 p_{12}(x)}{p_{11}(x)-p_{22}(x)}\right) & \text { if } p_{11} \neq p_{22} \\ \frac{\pi}{4} & \text { if } p_{11}=p_{22}\end{cases}
$$

then $Q$ takes the simpler form $Q=\left(\begin{array}{cc}q_{1} & 0 \\ 0 & q_{2}\end{array}\right)$ for some continuous functions $q_{1}$ and $q_{2}$. Thus (5) takes the canonical form

$$
\left.\begin{array}{l}
y_{2}^{\prime}+q_{1}(x) y_{1}=\lambda y_{1} \\
y_{1}^{\prime}-q_{2}(x) y_{2}=-\lambda y_{2}
\end{array}\right\} \quad(0<x<\infty)
$$

In the sequel we will be dealing mainly with the equivalent system

$$
\left.\begin{array}{l}
y_{1}^{\prime}-q_{2}(x) y_{2}=\lambda y_{2} \\
y_{2}^{\prime}+q_{1}(x) y_{1}=-\lambda y_{1}
\end{array}\right\} \quad(0<x<\infty)
$$


where $q_{1}$ and $q_{2}$ are continuous on $[0, \infty)$. Let

$$
\underline{\Phi}(x, \lambda)=\left(\begin{array}{l}
\phi_{1}(x, \lambda) \\
\phi_{2}(x, \lambda)
\end{array}\right) \quad \text { and } \quad \underline{\Theta}(x, \lambda)=\left(\begin{array}{c}
\theta_{1}(x, \lambda) \\
\theta_{2}(x, \lambda)
\end{array}\right)
$$

be the solutions of system (6) that satisfies

$$
\left.\left.\begin{array}{l}
\phi_{1}(0, \lambda)=-\cos \alpha \\
\phi_{2}(0, \lambda)=\sin \alpha
\end{array}\right\} \quad \text { and } \quad \begin{array}{l}
\theta_{1}(0, \lambda)=\sin \alpha \\
\theta_{2}(0, \lambda)=\cos \alpha
\end{array}\right\} \text {. }
$$

Let

$$
\underline{f}(x)=\left(\begin{array}{l}
f_{1}(x) \\
f_{2}(x)
\end{array}\right)
$$

be a vector-valued function such that $\underline{f} \in L_{2}\left(\mathbb{R}^{+}\right)$, i.e.

$$
\|\underline{f}\|_{L_{2}\left(\mathbb{R}^{+}\right)}^{2}=\int_{0}^{\infty}|\underline{f}(x)|^{2} d x=\int_{0}^{\infty}\left(\left|f_{1}(x)\right|^{2}+\left|f_{2}(x)\right|^{2}\right) d x<\infty .
$$

It is known [4] that for any non-real $\lambda$ there exists a function $m(\lambda)$, analytic in the upper and lower half-planes that are not necessarily analytic continuation of each other so that

$$
\underline{\Theta}(x, \lambda)+m(\lambda) \underline{\Phi}(x, \lambda),
$$

as a vector-valued function of $x$, is in $L_{2}\left(\mathbb{R}^{+}\right)$for non-real $\lambda$. Moreover,

$$
\frac{1}{\pi} \lim _{\delta \rightarrow 0+} \int_{\mu}^{\lambda} \Im m(u+i \delta) d u=\rho(\lambda)-\rho(\mu)
$$

where $\rho$ is a monotone increasing function (see [3]). If $\underline{f} \in L_{2}\left(\mathbb{R}^{+}\right)$, then

$$
F(\lambda)=\int_{0}^{\infty} \underline{f}^{T}(x) \underline{\Phi}(x) d x=\int_{0}^{\infty}\left(f_{1}(x) \phi_{1}(x, \lambda)+f_{2}(x) \phi_{2}(x, \lambda)\right) d x,
$$

the generalized Fourier transform of $\underline{f}$, belongs to $L_{2}(\mathbb{R}, d \rho)$ and the Parseval equality

$$
\int_{0}^{\infty}|\underline{f}(x)|^{2} d x=\int_{-\infty}^{\infty}|F(\lambda)|^{2} d \rho(\lambda)
$$

holds. In addition, if the integrals

$$
\int_{-\infty}^{\infty} F(\lambda) \phi_{1}(x, \lambda) d \rho(\lambda) \quad \text { and } \quad \int_{-\infty}^{\infty} F(\lambda) \phi_{2}(x, \lambda) d \rho(\lambda)
$$

converge absolutely and uniformly in $x$ in each finite interval, then

$$
\left.\begin{array}{l}
f_{1}(x)=\int_{-\infty}^{\infty} F(\lambda) \phi_{1}(x, \lambda) d \rho(\lambda) \\
f_{2}(x)=\int_{-\infty}^{\infty} F(\lambda) \phi_{2}(x, \lambda) d \rho(\lambda)
\end{array}\right\} .
$$


We also have the estimate

$$
\int_{-\infty}^{\infty} \frac{d \rho(\lambda)}{\lambda^{2}+1}<\infty
$$

Let us denote by $L$ the operator

$$
L=B \frac{d}{d x}-Q=\left(\begin{array}{cc}
0 & -1 \\
1 & 0
\end{array}\right) \frac{d}{d x}-\left(\begin{array}{cc}
q_{1}(x) & 0 \\
0 & q_{2}(x)
\end{array}\right)
$$

so that system (6) takes the form

$$
L Y=\lambda Y .
$$

Hence $\underline{\Phi}(x, \lambda)$ is a solution of the system

$$
L \underline{\Phi}=\lambda \underline{\Phi}
$$

and

$$
A^{T} \underline{\Phi}(0, \lambda)=0
$$

where

$$
A=\left(\begin{array}{c}
\sin \alpha \\
\cos \alpha
\end{array}\right) \quad \text { and } \quad \underline{\Phi}(0, \lambda)=\left(\begin{array}{c}
\phi_{1}(0, \lambda) \\
\phi_{2}(0, \lambda)
\end{array}\right) .
$$

We shall call a vector-valued function $\underline{f}$ the $\underline{\Phi}$-transform of $F$ if

$$
\underline{f}(x)=\int_{-\infty}^{\infty} F(\lambda) \underline{\Phi}(x, \lambda) d \rho(\lambda)
$$

where $\underline{\Phi}$ satisfies (12) and (13). We shall assume that $Q$ is infinitely differentiable, such that the spectrum of the system (12) is either $\mathbb{R}$ or $\mathbb{R}^{+}$.

\section{Paley-Wiener-type theorem}

The following lemma describes the image of the $\underline{\Phi}$-transform of a function $F$ such that $\lambda^{n} F(\lambda) \in L_{2}(\mathbb{R}, d \rho)$ for all $n \in \mathbb{N}_{0}$.

Lemma 1. Let the function $F$ be such that $\lambda^{n} F(\lambda) \in L_{2}(\mathbb{R}, d \rho)$ for all $n \in \mathbb{N}_{0}$. $A$ vector-valued function $\underline{f}$ is the $\underline{\Phi}$-transform of $F$ as given by (14) if and only if the following conditions are satisfied:

1) $\underline{f}$ is infinitely differentiable on $\mathbb{R}^{+}$.

2) $L^{n} \underline{f} \in L_{2}\left(\mathbb{R}^{+}\right)$for all $n$.

3) $\lim _{x \rightarrow 0} A^{T} \cdot\left(L^{n} \underline{f}\right)(x)=0$.

4) $\lim _{x \rightarrow \infty}\left(L^{n} \underline{f}\right)(x)=0$.

Proof . Necessity: Let $\lambda^{n} F(\lambda) \in L_{2}(\mathbb{R}, d \rho)$ for all $n \in \mathbb{N}_{0}$. It is easy to see that $\lambda^{n} F(\lambda) \in L_{1}(\mathbb{R}, d \rho)$. Indeed, applying formula (11) and the Cauchy-Schwartz inequality, we get

$$
\left(\int_{-\infty}^{\infty}\left|\lambda^{n} F(\lambda)\right| d \rho(\lambda)\right)^{2} \leq\left(\int_{-\infty}^{\infty}\left(\lambda^{2 n+2}+\lambda^{2 n}\right)|F(\lambda)|^{2} d \rho(\lambda)\right)\left(\int_{-\infty}^{\infty} \frac{d \rho(\lambda)}{\lambda^{2}+1}\right)<\infty .
$$


1) Since $Q$ is infinitely differentiable, so are $\phi_{1}(x, \lambda)$ and $\phi_{2}(x, \lambda)$ with respect to the variable $x$. It also follows as in the Sturm-Liouville case [7] that $\frac{\partial^{n} \phi_{1}(x, \lambda)}{\partial x^{n}}$ and $\frac{\partial^{n} \phi_{2}(x, \lambda)}{\partial x^{n}}$ are of order $\lambda^{n}$ as $\lambda \rightarrow \infty$. Therefore,

$$
\underline{f}^{(n)}(x)=\int_{-\infty}^{\infty} F(\lambda) \frac{\partial^{n} \underline{\Phi}(x, \lambda)}{\partial x^{n}} d \rho(\lambda)
$$

exists for all $n$, where

$$
\underline{f}^{(n)}(x)=\left(\begin{array}{l}
f_{1}^{(n)}(x) \\
f_{2}^{(n)}(x)
\end{array}\right) .
$$

2) By applying the differential operator $L$ to (14) $n$ times, we have

$$
\left(L^{n} \underline{f}\right)(x)=\int_{-\infty}^{\infty} F(\lambda) L^{n} \underline{\Phi}(x, \lambda) d \rho(\lambda)=\int_{-\infty}^{\infty} \lambda^{n} F(\lambda) \underline{\Phi}(x, \lambda) d \rho(\lambda),
$$

and since $\lambda^{n} F(\lambda) \in L_{2}(\mathbb{R}, d \rho)$ we have $L^{n} \underline{f} \in L_{2}\left(\mathbb{R}^{+}\right)$.

3) By taking the matrix product with $A^{T}$, we get

$$
\begin{aligned}
\lim _{x \rightarrow 0+} A^{T} \cdot\left(L^{n} \underline{f}\right)(x) & =\lim _{x \rightarrow 0+} \int_{-\infty}^{\infty} \lambda^{n} F(\lambda)\left(A^{T} \cdot \underline{\Phi}(x, \lambda)\right) d \rho(\lambda) \\
& =\int_{-\infty}^{\infty} \lambda^{n} F(\lambda)\left(A^{T} \cdot \underline{\Phi}(0, \lambda)\right) d \rho(\lambda) \\
& =0 .
\end{aligned}
$$

Taking the limit inside the integral is justified by the Lebesgue dominated convergence theorem.

4) Let

$$
\underline{\tilde{f}}(x)=B \overline{\bar{f}}(x)=\left(\begin{array}{cc}
0 & -1 \\
1 & 0
\end{array}\right)\left(\begin{array}{l}
\overline{f_{1}}(x) \\
\overline{f_{2}}(x)
\end{array}\right)=\left(\begin{array}{c}
-\overline{f_{2}}(x) \\
\overline{f_{1}}(x)
\end{array}\right) .
$$

We have

$$
\begin{aligned}
\int_{0}^{N} & \underline{\tilde{f}}^{T}(x) \cdot L \underline{f}(x) d x \\
= & \int_{0}^{N}\left\{\bar{f}_{2}(x)\left[f_{2}^{\prime}(x)+q_{1}(x) f_{1}(x)\right]+\bar{f}_{1}(x)\left[f_{1}^{\prime}(x)-q_{2}(x) f_{2}(x)\right]\right\} d x \\
= & {\left.\left[\left|f_{1}(x)\right|^{2}+\left|f_{2}(x)\right|^{2}\right]\right|_{0} ^{N} } \\
& +\int_{0}^{N}\left\{\left[-\bar{f}_{1}^{\prime}(x)+\bar{f}_{2}(x) q_{1}(x)\right] f_{1}(x)+\left[-\bar{f}_{2}^{\prime}(x)-\bar{f}_{1}(x) q_{2}(x)\right] f_{2}(x)\right\} d x \\
= & {\left.\left[\left|f_{1}(x)\right|^{2}+\left|f_{2}(x)\right|^{2}\right]\right|_{0} ^{N}+\int_{0}^{N} \underline{f}^{T}(x) \cdot L \underline{\tilde{f}}(x) d x }
\end{aligned}
$$

Hence

$$
\begin{aligned}
& \left|f_{1}(N)\right|^{2}+\left|f_{2}(N)\right|^{2} \\
& \quad=\left|f_{1}(0)\right|^{2}+\left|f_{2}(0)\right|^{2}+\int_{0}^{N} \underline{\tilde{f}}^{T}(x) \cdot L \underline{f}(x) d x-\int_{0}^{N} \underline{f}^{T}(x) \cdot L \underline{\tilde{f}}(x) d x .
\end{aligned}
$$


Since $\underline{f}$ and $L \underline{f}$ are in $L_{2}\left(\mathbb{R}^{+}\right)$, it follows that $\underline{\tilde{f}}$ and $L \underline{\tilde{f}}$ are also in $L_{2}\left(\mathbb{R}^{+}\right)$. Therefore, the right-hand side of (15) approaches a limit as $N \rightarrow \infty$; hence so is the left-hand side of (15). But because $f_{1}$ and $f_{2}$ belong to $L_{2}\left(\mathbb{R}^{+}\right)$, the limit must be zero, i.e.

$$
\lim _{N \rightarrow \infty} f_{1}(N)=\lim _{N \rightarrow \infty} f_{2}(N)=0 .
$$

Similarly one can show that $\lim _{x \rightarrow \infty}\left(L^{n} f\right)(x)=0$ for all $n$.

Sufficiency: Let $\underline{f}$ satisfy conditions 1 - 4 of the lemma. Since $\underline{f} \in L_{2}\left(\mathbb{R}^{+}\right)$(condition 1 with $n=0)$, there exists $F \in L_{2}(\mathbb{R}, d \rho)$ such that

$$
F(\lambda)=\int_{0}^{\infty} \underline{f}^{T}(x) \cdot \underline{\Phi}(x, \lambda) d x .
$$

Then

$$
\lambda^{n} F(\lambda)=\int_{0}^{\infty} \lambda^{n} \underline{f}^{T}(x) \cdot \underline{\Phi}(x, \lambda) d x=\int_{0}^{\infty} \underline{f}^{T}(x) \cdot L^{n} \underline{\Phi}(x, \lambda) d x .
$$

We show by induction on $n$ that

$$
\lambda^{n} F(\lambda)=\int_{0}^{\infty}\left(L^{n} \underline{f}\right)^{T}(x) \cdot \underline{\Phi}(x, \lambda) d x .
$$

For $n=1$,

$$
\begin{aligned}
\lambda F(\lambda)= & \int_{0}^{\infty} \underline{f}^{T}(x) \cdot L \underline{\Phi}(x, \lambda) d x \\
= & \int_{0}^{\infty}\left\{-f_{1}(x)\left[\phi_{2}^{\prime}(x, \lambda)+q_{1}(x) \phi_{1}(x, \lambda)\right]\right. \\
& \left.+f_{2}(x)\left[\phi_{1}^{\prime}(x, \lambda)-q_{2}(x) \phi_{2}(x, \lambda)\right]\right\} d x \\
= & {\left.\left[-f_{1}(x) \phi_{2}(x, \lambda)+f_{2}(x) \phi_{1}(x, \lambda)\right]\right|_{0} ^{\infty} } \\
& +\int_{0}^{\infty}\left\{\left[-\phi_{1}(x, \lambda) f_{2}^{\prime}(x)+\phi_{2}(x, \lambda) f_{1}^{\prime}(x)\right]\right. \\
& \left.-\left[\phi_{1}(x, \lambda) f_{1}(x) q_{1}(x)+\phi_{2}(x, \lambda) f_{2}(x) q_{2}(x)\right]\right\} d x \\
= & {\left.\left[-f_{1}(x) \phi_{2}(x, \lambda)+f_{2}(x) \phi_{1}(x, \lambda)\right]\right|_{0} ^{\infty} } \\
& +\int_{0}^{\infty}\left\{\left[-f_{2}^{\prime}(x)-f_{1}(x) q_{1}(x)\right] \phi_{1}(x, \lambda)\right. \\
& \left.+\left[f_{1}^{\prime}(x)-f_{2}(x) q_{2}(x)\right] \phi_{2}(x, \lambda)\right\} d x \\
= & {\left.\left[-f_{1}(x) \phi_{2}(x, \lambda)+f_{2}(x) \phi_{1}(x, \lambda)\right]\right|_{0} ^{\infty} } \\
& +\int_{0}^{\infty}[(L \underline{f})(x)]^{T} \cdot \underline{\Phi}(x, \lambda) d x .
\end{aligned}
$$

Since $f(x)$ and $\underline{\Phi}(x, \lambda)$ satisfy the same initial condition (13) it follows that

$$
f_{1}(0) \phi_{2}(0, \lambda)-f_{2}(0) \phi_{1}(0, \lambda)=0 .
$$


As $x \rightarrow \infty$, from condition $4(n=0)$ it follows that $\lim _{x \rightarrow \infty} \underline{f}(x)=0$, and because $\underline{\Phi}(x, \lambda)$ is bounded in $x$ for each fixed $\lambda$ we have

$$
\lim _{x \rightarrow \infty}\left[f_{1}(x) \phi_{2}(x, \lambda)-f_{2}(x) \phi_{1}(x, \lambda)\right]=0
$$

Hence,

$$
\lambda F(\lambda)=\int_{0}^{\infty}[(L \underline{f})(x)]^{T} \cdot \underline{\Phi}(x, \lambda) d x .
$$

As $L \underline{f} \in L_{2}\left(\mathbb{R}^{+}\right)$, we have $\lambda F(\lambda) \in L_{2}(R, d \rho)$.

Now assume that

$$
\lambda^{n} F(\lambda)=\int_{0}^{\infty}\left[\left(L^{n} \underline{f}\right)(x)\right]^{T} \cdot \underline{\Phi}(x, \lambda) d x .
$$

Then

$$
\begin{aligned}
\lambda^{n+1} F(\lambda) & =\int_{0}^{\infty}\left[\left(L^{n} \underline{f}\right)(x)\right]^{T} \cdot L \underline{\Phi}(x, \lambda) d x \\
& =\left.\left[-g_{1}(x) \phi_{2}(x, \lambda)+g_{2}(x) \phi_{1}(x, \lambda)\right]\right|_{0} ^{\infty}+\int_{0}^{\infty}[(L \underline{g})(x)]^{T} \cdot \underline{\Phi}(x, \lambda) d x
\end{aligned}
$$

where

$$
\underline{g}(x)=\left(\begin{array}{l}
g_{1}(x) \\
g_{2}(x)
\end{array}\right)=\left(L^{n} \underline{f}\right)(x) .
$$

For $\underline{g}(x)$ and $\underline{\Phi}(x, \lambda)$ satisfy the same initial condition (13), then by conditions 3 and 4 we have

$$
\left.\left[-g_{1}(x) \phi_{2}(x, \lambda)+g_{2}(x) \phi_{1}(x, \lambda)\right]\right|_{0} ^{\infty}=0
$$

Thus,

$$
\lambda^{n+1} F(\lambda)=\int_{0}^{\infty}\left[\left(L^{n+1} \underline{f}\right)(x)\right]^{T} \cdot \underline{\Phi}(x, \lambda) d x
$$

and since $L^{n+1} f \in L_{2}\left(\mathbb{R}^{+}\right)$, it follows that $\lambda^{n+1} F(\lambda) \in L_{2}(\mathbb{R}, d \rho)$

Lemma 2. Let $\underline{f}$ be the $\underline{\Phi}$-transform of a function $F$ as given by $(14)$. Let $\lambda^{n} F(\lambda) \in$ $L_{2}(\mathbb{R}, d \rho)$ for $n \in \mathbb{N}_{0}^{-}$. Then

$$
\lim _{n \rightarrow \infty}\left\|L^{n} \underline{f}\right\|_{L_{2}\left(\mathbb{R}^{+}\right)}^{\frac{1}{n}}=\sup _{\lambda \in \operatorname{supp} F}|\lambda|
$$

Proof. From the relation

$$
\left(L^{n} \underline{f}\right)(x)=\int_{-\infty}^{\infty} \lambda^{n} F(\lambda) \underline{\Phi}(x, \lambda) d \rho(\lambda)
$$

and the Parseval equation (9) we have

$$
\left\|L^{n} \underline{f}\right\|_{L_{2}\left(\mathbb{R}^{+}\right)}^{2}=\int_{-\infty}^{\infty} \lambda^{2 n}|F(\lambda)|^{2} d \rho(\lambda)
$$


First, let $F$ have a compact support: $\sup _{\lambda \in \operatorname{supp} F}|\lambda|=\delta<\infty$. Then

$$
\int_{-\infty}^{\infty} \lambda^{2 n}|F(\lambda)|^{2} d \rho(\lambda)=\int_{-\delta}^{\delta} \lambda^{2 n}|F(\lambda)|^{2} d \rho(\lambda) \leq \delta^{2 n} \int_{-\delta}^{\delta}|F(\lambda)|^{2} d \rho(\lambda) .
$$

Hence,

$$
\limsup _{n \rightarrow \infty}\left\|L^{n} \underline{f}\right\|_{L_{2}\left(\mathbb{R}^{+}\right)}^{\frac{1}{n}} \leq \delta \limsup _{n \rightarrow \infty}\left\{\int_{-\delta}^{\delta}|F(\lambda)|^{2} d \rho(\lambda)\right\}^{\frac{1}{2 n}}=\delta .
$$

On the other hand, because $\delta$ is the supremum of the support of $F$, for any $\varepsilon$ with $0<\varepsilon<\delta$ we have

$$
\int_{\delta-\varepsilon<|\lambda|<\delta}|F(\lambda)|^{2} d \rho(\lambda)>0
$$

Therefore,

$$
\begin{aligned}
\liminf _{n \rightarrow \infty}\left\|L^{n} \underline{f}\right\|_{L_{2}\left(\mathbb{R}^{+}\right)}^{\frac{1}{n}} & \geq \liminf _{n \rightarrow \infty}\left\{\int_{\delta-\varepsilon<|\lambda|<\delta} \lambda^{2 n}|F(\lambda)|^{2} d \rho(\lambda)\right\}^{\frac{1}{2 n}} \\
& \geq(\delta-\varepsilon) \liminf _{n \rightarrow \infty}\left\{\int_{\delta-\varepsilon<|\lambda|<\delta}|F(\lambda)|^{2} d \rho(\lambda)\right\}^{\frac{1}{2 n}} \\
& =\delta-\varepsilon .
\end{aligned}
$$

Because $\varepsilon>0$ is arbitrary, we obtain

$$
\lim _{n \rightarrow \infty}\left\|L^{n} \underline{f}\right\|_{L_{2}\left(\mathbb{R}^{+}\right)}^{\frac{1}{n}}=\delta .
$$

Now let $F$ have a unbounded support. Then for any $N$ large enough

$$
\int_{|\lambda|>N}|F(\lambda)|^{2} d \rho(\lambda)>0 .
$$

Consequently,

$$
\begin{aligned}
\lim _{n \rightarrow \infty}\left\|L^{n} \underline{f}\right\|_{L_{2}\left(\mathbb{R}^{+}\right)}^{\frac{1}{n}} & \geq \lim _{n \rightarrow \infty}\left\{\int_{|\lambda|>N} \lambda^{2 n}|F(\lambda)|^{2} d \rho(\lambda)\right\}^{\frac{1}{2 n}} \\
& \geq N \lim _{n \rightarrow \infty}\left\{\int_{|\lambda|>N}|F(\lambda)|^{2} d \rho(\lambda)\right\}^{\frac{1}{2 n}} \\
& =N .
\end{aligned}
$$

Because $N$ is arbitrary, we obtain $\lim _{n \rightarrow \infty}\left\|L^{n} \underline{f}\right\|_{L_{2}\left(\mathbb{R}^{+}\right)}^{\frac{1}{n}}=\infty$

Now we are ready to state and prove the following Paley-Wiener-type theorem. 
Theorem. A vector-valued function $f$ is the $\underline{\Phi}$-transform (14) of a function $F \in$ $L_{2}(\mathbb{R}, d \rho)$ with compact support if and only if $\underline{f}$ satisfies conditions $1-4$ of Lemma 1 and

$$
\lim _{n \rightarrow \infty}\left\|L^{n} \underline{f}\right\|_{L_{2}\left(\mathbb{R}^{+}\right)}^{\frac{1}{n}}<\infty .
$$

Proof. Let $F \in L_{2}(\mathbb{R}, d \rho)$ have a compact support. Then $\lambda^{n} F(\lambda) \in L_{2}(\mathbb{R}, d \rho)$ for all $n \in \mathbb{N}_{0}$. Consequently, by Lemma $1, \underline{f}$ satisfies conditions 1 - 4 of Lemma 1 , and by Lemma 2

$$
\lim _{n \rightarrow \infty}\left\|L^{n} \underline{f}\right\|_{L_{2}\left(\mathbb{R}^{+}\right)}^{\frac{1}{n}}=\sup _{\lambda \in \operatorname{supp} F}|\lambda|<\infty .
$$

Conversely, let $f$ satisfy conditions 1 - 4 of Lemma 1 and (16). By Lemma $1, f$ is the

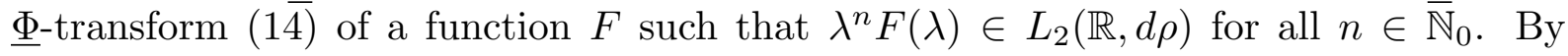
Lemma 2 and equation (16) we have

$$
\sup _{\lambda \in \operatorname{supp} F}|\lambda|=\lim _{n \rightarrow \infty}\left\|L^{n} \underline{f}\right\|_{L_{2}\left(\mathbb{R}^{+}\right)}^{\frac{1}{n}}<\infty .
$$

Hence, $F$ has a compact support

\section{Examples}

As examples, the Hartley and some transforms with the Airy and the Bessel functions as kernels are derived, and the Paley-Wiener theorems are obtained for these transforms.

Example 1 (Fourier and Hartley transform). Consider the boundary-value problem (6) - (8) in which $q_{1}=0=q_{2}$. It is easy to see that

$$
\underline{\Phi}(x, \lambda)=\left(\begin{array}{c}
-\cos (\lambda x+\alpha) \\
\sin (\lambda x+\alpha)
\end{array}\right) \quad \text { and } \quad \underline{\Theta}(x, \lambda)=\left(\begin{array}{c}
\sin (\lambda x+\alpha) \\
\cos (\lambda x+\alpha)
\end{array}\right)
$$

and $m(\lambda)=i$, since

$$
\underline{\Theta}(x, \lambda)+m(\lambda) \underline{\Phi}(x, \lambda)=\left(\begin{array}{c}
-i e^{i(\lambda x+\alpha)} \\
e^{i(\lambda x+\alpha)}
\end{array}\right) \in L_{2}\left(\mathbb{R}^{+}\right)
$$

for $\Im \lambda>0$. Thus $d \rho(\lambda)=\frac{1}{\pi} d \lambda$,

$$
F(\lambda)=\int_{0}^{\infty}\left\{-\cos (\lambda x+\alpha) f_{1}(x)+\sin (\lambda x+\alpha) f_{2}(x)\right\} d x
$$

and

$$
\left.\begin{array}{l}
f_{1}(x)=-\frac{1}{\pi} \int_{-\infty}^{\infty} \cos (\lambda x+\alpha) F(\lambda) d \lambda \\
f_{2}(x)=\frac{1}{\pi} \int_{-\infty}^{\infty} \sin (\lambda x+\alpha) F(\lambda) d \lambda
\end{array}\right\} .
$$

The Paley-Wiener-type theorem for the transforms (17) takes the following form. 
Corollary 1. A vector-valued function $f$ is the $\underline{\Phi}$-transform (17) of a function $F \in L_{2}(R)$ with compact support if and only if $\underline{f}$ satisfies the following conditions:

1) $\underline{f}$ is infinitely differentiable on $\mathbb{R}^{+}$.

2) $\left(\begin{array}{cc}0 & -\frac{d}{d x} \\ \frac{d}{d x} & 0\end{array}\right)^{n} \underline{f}(x) \in L_{2}\left(R^{+}\right)$for all $n$.

3) $\lim _{x \rightarrow 0}(\sin \alpha, \cos \alpha) \cdot\left(\begin{array}{cc}0 & -\frac{d}{d x} \\ \frac{d}{d x} & 0\end{array}\right)^{n} \underline{f}(x)=0$.

4) $\lim _{x \rightarrow \infty}\left(\begin{array}{cc}0 & -\frac{d}{d x} \\ \frac{d}{d x} & 0\end{array}\right)^{n} \underline{f}(x)=0$.

5) $\lim _{n \rightarrow \infty}\left\|\left(\begin{array}{cc}0 & -\frac{d}{d x} \\ -\frac{d}{d x} & 0\end{array}\right)^{n} \underline{f}(x)\right\|_{L_{2}\left(\mathbb{R}^{+}\right)}^{\frac{1}{n}}<\infty$.

Let $\alpha=0$ and

$$
2 f(x)=-f_{1}(|x|)+i \operatorname{sign} x f_{2}(|x|) \quad(x \in \mathbb{R}) .
$$

Then we obtain the pair of Fourier transforms

$$
\left.\begin{array}{rl}
F(\lambda) & =\int_{-\infty}^{\infty} e^{-i \lambda x} f(x) d x \\
f(x) & =\frac{1}{2 \pi} \int_{-\infty}^{\infty} e^{i \lambda x} F(\lambda) d \lambda
\end{array}\right\}
$$

If we put $\alpha=0$ and

$$
2 f(x)=-f_{1}(|x|)+\operatorname{sign} x f_{2}(|x|) \quad(x \in R),
$$

then we arrive at the pair of Hartley transforms

$$
\left.\begin{array}{rl}
F(\lambda) & =\int_{-\infty}^{\infty}(\cos \lambda x+\sin \lambda x) f(x) d x \\
f(x) & =\frac{1}{2 \pi} \int_{-\infty}^{\infty}(\cos \lambda x+\sin \lambda x) F(\lambda) d \lambda
\end{array}\right\} .
$$

Example 2 (Airy transform). Consider system (6) - (8) with $q_{1}=0, q_{2}(x)=-x$ and $\alpha=0$ :

$$
\left.\begin{array}{rl}
y_{1}^{\prime}+x y_{2} & =\lambda y_{2} \\
y_{2}^{\prime} & =-\lambda y_{1}
\end{array}\right\} \quad(0<x<\infty) .
$$

Eliminating $y_{1}$ from this system yields

$$
y_{2}^{\prime \prime}-\left(\lambda x-\lambda^{2}\right) y_{2}=0
$$

Setting $t+\tilde{\lambda}=\lambda^{\frac{4}{3}}-\lambda^{\frac{1}{3}} x$ we obtain

$$
\frac{d^{2} y_{2}}{d t^{2}}+(t+\tilde{\lambda}) y_{2}=0 \quad \text { and } \quad y_{1}=\lambda^{-\frac{2}{3}} \frac{d y_{2}}{d t}
$$


The general solution of the first equation is

$$
y_{2}(t)=A(t+\tilde{\lambda})^{\frac{1}{2}} J_{\frac{1}{3}}(X)+B(t+\tilde{\lambda})^{\frac{1}{2}} Y_{\frac{1}{3}}(X)
$$

where

$$
X=\frac{2}{3}(t+\tilde{\lambda})^{\frac{3}{2}}=\frac{2}{3} \lambda^{\frac{1}{2}}(\lambda-x)^{\frac{3}{2}}
$$

and $J_{\mu}$ and $Y_{\mu}$ are the Bessel functions of the first and second kinds, respectively. Hence

$$
y_{2}(x)=\lambda^{\frac{1}{6}}\left\{A(\lambda-x)^{\frac{1}{2}} J_{\frac{1}{3}}(X)+B(\lambda-x)^{\frac{1}{2}} Y_{\frac{1}{3}}(X)\right\}
$$

and

$$
\begin{aligned}
y_{1}(x)= & \lambda^{-\frac{5}{6}}\left\{A \lambda^{\frac{1}{2}}(\lambda-x) J_{\frac{1}{3}}^{\prime}(X)+\frac{A}{2 \sqrt{\lambda-x}} J_{\frac{1}{3}}(X)\right. \\
& \left.+B \lambda^{\frac{1}{2}}(\lambda-x) Y_{\frac{1}{3}}^{\prime}(X)+\frac{B}{2 \sqrt{\lambda-x}} Y_{\frac{1}{3}}(X)\right\} .
\end{aligned}
$$

Since $X=\frac{2}{3} \lambda^{2}$ at $x=0$ we have

$$
y_{2}(0)=A \lambda^{\frac{2}{3}} J_{\frac{1}{3}}\left(\frac{2}{3} \lambda^{2}\right)+B \lambda^{\frac{2}{3}} Y_{\frac{1}{3}}\left(\frac{2}{3} \lambda^{2}\right)=0
$$

and

$$
\begin{aligned}
y_{1}(0)= & \frac{A}{\lambda^{\frac{5}{6}}}\left\{\lambda^{\frac{3}{2}} J_{\frac{1}{3}}^{\prime}\left(\frac{2}{3} \lambda^{2}\right)+\frac{1}{2 \sqrt{\lambda}} J_{\frac{1}{3}}\left(\frac{2}{3} \lambda^{2}\right)\right\} \\
& +\frac{B}{\lambda^{\frac{5}{6}}}\left\{\lambda^{\frac{3}{2}} Y_{\frac{1}{3}}^{\prime}\left(\frac{2}{3} \lambda^{2}\right)+\frac{1}{2 \sqrt{\lambda}} Y_{\frac{1}{3}}\left(\frac{2}{3} \lambda^{2}\right)\right\}=1
\end{aligned} .
$$

Solving this system for $A$ and $B$, we obtain $\phi_{1}$ and $\phi_{2}$. The explicit solution for $\phi_{1}$ and $\phi_{2}$ can be found in [7: pp. 92 - 93] and therefore the kernels of the integrals in (10) can be expressed in terms of the Bessel functions $J_{\mu}$ and $Y_{\mu}$.

However, since the argument of the Bessel functions,

$$
X=\frac{2}{3} \lambda^{\frac{1}{2}}(\lambda-x)^{\frac{3}{2}}
$$

may be complex or real depending on whether $x$ is greater or less than $\lambda$, and hence the integrals have to be split into different regions with different kinds of Bessel functions as kernels, it is more convenient to use the Airy functions. In so doing, we can express $f_{1}$ and $f_{2}$ as single integrals extending over the whole real line (see (23) below).

So, let us make the change of variable $\lambda x-\lambda^{2}=\lambda^{\frac{2}{3}} z$ to obtain the Airy differential equation

$$
\frac{d^{2} y_{2}}{d z^{2}}-z y_{2}=0
$$

whose general solution is

$$
y_{2}=a \operatorname{Ai}(z)+b \operatorname{Bi}(z)
$$


Here $\mathrm{Ai}$ and $\mathrm{Bi}$ are the Airy functions [1]. Hence,

$$
y_{2}(x, \lambda)=a \operatorname{Ai}\left(\lambda^{\frac{1}{3}} x-\lambda^{\frac{4}{3}}\right)+b \operatorname{Bi}\left(\lambda^{\frac{1}{3}} x-\lambda^{\frac{4}{3}}\right) .
$$

Since $y_{1}=-\lambda^{-1} y_{2}^{\prime}$ we have

$$
y_{1}(x, \lambda)=-a \lambda^{-\frac{2}{3}} \operatorname{Ai}^{\prime}\left(\lambda^{\frac{1}{3}} x-\lambda^{\frac{4}{3}}\right)-b \lambda^{-\frac{2}{3}} \operatorname{Bi}^{\prime}\left(\lambda^{\frac{1}{3}} x-\lambda^{\frac{4}{3}}\right) .
$$

Thus, (18) and (19) are the general solution of system (18). The solution $\underline{\Phi}(x, \lambda)$ satisfies the initial condition

$$
\left.\begin{array}{l}
\phi_{1}(0, \lambda)=-1 \\
\phi_{2}(0, \lambda)=0
\end{array}\right\}
$$

Thus

$$
\left.\begin{array}{r}
a \mathrm{Ai}\left(-\lambda^{\frac{4}{3}}\right)+b \operatorname{Bi}\left(-\lambda^{\frac{4}{3}}\right)=0 \\
a \lambda^{-\frac{2}{3}} \operatorname{Ai}^{\prime}\left(-\lambda^{\frac{4}{3}}\right)+b \lambda^{-\frac{2}{3}} \operatorname{Bi}^{\prime}\left(-\lambda^{\frac{4}{3}}\right)=1
\end{array}\right\} .
$$

Using the Wronskian equality for the Airy functions [1]

$$
W(\operatorname{Ai}(x), \operatorname{Bi}(x)):=\operatorname{Ai}(x) \operatorname{Bi}^{\prime}(x)-\operatorname{Ai}^{\prime}(x) \operatorname{Bi}(x)=\frac{1}{\pi}
$$

we get

$$
\left.\begin{array}{l}
a=-\pi \lambda^{\frac{2}{3}} \operatorname{Bi}\left(-\lambda^{\frac{4}{3}}\right) \\
b=\pi \lambda^{\frac{2}{3}} \operatorname{Ai}\left(-\lambda^{\frac{4}{3}}\right)
\end{array}\right\}
$$

Thus,

$$
\left.\begin{array}{l}
\phi_{1}(x, \lambda)=\pi \operatorname{Bi}\left(-\lambda^{\frac{4}{3}}\right) \operatorname{Ai}^{\prime}\left(\lambda^{\frac{1}{3}} x-\lambda^{\frac{4}{3}}\right)-\pi \operatorname{Ai}\left(-\lambda^{\frac{4}{3}}\right) \operatorname{Bi}^{\prime}\left(\lambda^{\frac{1}{3}} x-\lambda^{\frac{4}{3}}\right) \\
\phi_{2}(x, \lambda)=-\pi \lambda^{\frac{2}{3}} \operatorname{Bi}\left(-\lambda^{\frac{4}{3}}\right) \operatorname{Ai}\left(\lambda^{\frac{1}{3}} x-\lambda^{\frac{4}{3}}\right)+\pi \lambda^{\frac{2}{3}} \operatorname{Ai}\left(-\lambda^{\frac{4}{3}}\right) \operatorname{Bi}\left(\lambda^{\frac{1}{3}} x-\lambda^{\frac{4}{3}}\right)
\end{array}\right\} .
$$

Similarly, the solution $\underline{\Theta}(x, \lambda)$ satisfies the initial condition

$$
\left.\begin{array}{l}
\theta_{1}(0, \lambda)=0 \\
\theta_{2}(0, \lambda)=1
\end{array}\right\}
$$

that yields

$$
\left.\begin{array}{r}
a \operatorname{Ai}\left(-\lambda^{\frac{4}{3}}\right)+b \operatorname{Bi}\left(-\lambda^{\frac{4}{3}}\right)=1 \\
a \lambda^{-\frac{2}{3}} \operatorname{Ai}^{\prime}\left(-\lambda^{\frac{4}{3}}\right)+b \lambda^{-\frac{2}{3}} \operatorname{Bi}^{\prime}\left(-\lambda^{\frac{4}{3}}\right)=0
\end{array}\right\} .
$$

Solving this system we get

$$
\left.\begin{array}{l}
a=\pi \mathrm{Bi}^{\prime}\left(-\lambda^{\frac{4}{3}}\right) \\
b=-\pi \mathrm{Ai}^{\prime}\left(-\lambda^{\frac{4}{3}}\right)
\end{array}\right\} .
$$

Thus

$$
\left.\begin{array}{l}
\theta_{1}(x, \lambda)=-\pi \lambda^{-\frac{2}{3}} \operatorname{Bi}^{\prime}\left(-\lambda^{\frac{4}{3}}\right) \operatorname{Ai}^{\prime}\left(\lambda^{\frac{1}{3}} x-\lambda^{\frac{4}{3}}\right)+\pi \lambda^{-\frac{2}{3}} \operatorname{Ai}^{\prime}\left(-\lambda^{\frac{4}{3}}\right) \operatorname{Bi}^{\prime}\left(\lambda^{\frac{1}{3}} x-\lambda^{\frac{4}{3}}\right) \\
\theta_{2}(x, \lambda)=\pi \operatorname{Bi}^{\prime}\left(-\lambda^{\frac{4}{3}}\right) \operatorname{Ai}\left(\lambda^{\frac{1}{3}} x-\lambda^{\frac{4}{3}}\right)-\pi \operatorname{Ai}^{\prime}\left(-\lambda^{\frac{4}{3}}\right) \operatorname{Bi}\left(\lambda^{\frac{1}{3}} x-\lambda^{\frac{4}{3}}\right)
\end{array}\right\} .
$$


We have

$$
\begin{aligned}
\theta_{1}(x, \lambda)+m(\lambda) \phi_{1}(x, \lambda)= & {\left[-\lambda^{-\frac{2}{3}} \operatorname{Bi}^{\prime}\left(-\lambda^{\frac{4}{3}}\right)+m(\lambda) \operatorname{Bi}\left(-\lambda^{\frac{4}{3}}\right)\right] \pi \operatorname{Ai}^{\prime}\left(\lambda^{\frac{1}{3}} x-\lambda^{\frac{4}{3}}\right) } \\
& +\left[\lambda^{-\frac{2}{3}} \operatorname{Ai}^{\prime}\left(-\lambda^{\frac{4}{3}}\right)-m(\lambda) \operatorname{Ai}\left(-\lambda^{\frac{4}{3}}\right)\right] \pi \operatorname{Bi}^{\prime}\left(\lambda^{\frac{1}{3}} x-\lambda^{\frac{4}{3}}\right) \\
\theta_{2}(x, \lambda)+m(\lambda) \phi_{2}(x, \lambda)= & {\left[\operatorname{Bi}^{\prime}\left(-\lambda^{\frac{4}{3}}\right)-m(\lambda) \lambda^{\frac{2}{3}} \operatorname{Bi}\left(-\lambda^{\frac{4}{3}}\right)\right] \pi \operatorname{Ai}\left(\lambda^{-\frac{1}{3}} x-\lambda^{\frac{4}{3}}\right) } \\
& +\left[-\operatorname{Ai}^{\prime}\left(-\lambda^{\frac{4}{3}}\right)+m(\lambda) \lambda^{\frac{2}{3}} \operatorname{Ai}\left(-\lambda^{\frac{4}{3}}\right)\right] \pi \operatorname{Bi}\left(\lambda^{\frac{1}{3}} x-\lambda^{\frac{4}{3}}\right) .
\end{aligned}
$$

If we take

$$
m(\lambda)=\lambda^{-\frac{2}{3}} \frac{\operatorname{Ai}^{\prime}\left(-\lambda^{\frac{4}{3}}\right)+i \operatorname{Bi}^{\prime}\left(-\lambda^{\frac{4}{3}}\right)}{\operatorname{Ai}\left(-\lambda^{\frac{4}{3}}\right)+i \operatorname{Bi}\left(-\lambda^{\frac{4}{3}}\right)},
$$

then

$$
\left.\begin{array}{l}
\theta_{1}(x, \lambda)+m(\lambda) \phi_{1}(x, \lambda)=-\lambda^{-\frac{2}{3}} \frac{\operatorname{Ai}^{\prime}\left(\lambda^{\frac{1}{3}} x-\lambda^{\frac{4}{3}}\right)+i \operatorname{Bi}^{\prime}\left(\lambda^{\frac{1}{3}} x-\lambda^{\frac{4}{3}}\right)}{\operatorname{Ai}\left(-\lambda^{\frac{4}{3}}\right)+i \operatorname{Bi}\left(-\lambda^{\frac{4}{3}}\right)} \\
\theta_{2}(x, \lambda)+m(\lambda) \phi_{2}(x, \lambda)=\frac{\operatorname{Ai}\left(\lambda^{\frac{1}{3}} x-\lambda^{\frac{4}{3}}\right)+i \operatorname{Bi}\left(\lambda^{\frac{1}{3}} x-\lambda^{\frac{4}{3}}\right)}{\operatorname{Ai}\left(-\lambda^{\frac{4}{3}}\right)+i \operatorname{Bi}\left(-\lambda^{\frac{4}{3}}\right)}
\end{array}\right\} .
$$

The relations between the Airy functions and the Hankel functions $H_{\nu}^{(2)}{ }^{[1]}$

$$
\left.\begin{array}{l}
H_{-\frac{1}{3}}^{(2)}\left(\frac{2}{3} z^{\frac{3}{2}}\right)=e^{-i \frac{\pi}{6}} \sqrt{\frac{3}{z}}(\operatorname{Ai}(-z)+i \operatorname{Bi}(-z)) \\
H_{\frac{2}{3}}^{(2)}\left(\frac{2}{3} z^{\frac{3}{2}}\right)=e^{-i \frac{\pi}{6}} \frac{\sqrt{3}}{z}\left(\operatorname{Ai}^{\prime}(-z)+i \operatorname{Bi}^{\prime}(-z)\right)
\end{array}\right\}
$$

yield

$$
\left.\begin{array}{l}
\theta_{1}(x, \lambda)+m(\lambda) \phi_{1}(x, \lambda)=-\frac{(\lambda-x) H_{\frac{2}{3}}^{(2)}\left(\frac{2}{3} \lambda^{\frac{1}{2}}(\lambda-x)^{\frac{3}{2}}\right)}{\lambda H_{-\frac{1}{3}}^{(2)}\left(\frac{2}{3} \lambda^{2}\right)} \\
\theta_{2}(x, \lambda)+m(\lambda) \phi_{2}(x, \lambda)=\frac{(\lambda-x)^{\frac{1}{2}} H_{-\frac{1}{3}}^{(2)}\left(\frac{2}{3} \lambda^{\frac{1}{2}}(\lambda-x)^{\frac{3}{2}}\right)}{\lambda^{\frac{1}{2}} H_{-\frac{1}{3}}^{(2)}\left(\frac{2}{3} \lambda^{2}\right)}
\end{array}\right\} .
$$

Using the asymptotic expansion for the Hankel function [1]

$$
H_{\nu}^{(2)}(z)=\sqrt{\frac{2}{\pi z}} e^{-i\left(z-\frac{\nu}{2} \pi-\frac{\pi}{4}\right)}\left(1+O\left(\frac{1}{z}\right)\right)
$$

as $z \rightarrow \infty \quad(-2 \pi<\arg z<\pi)$, it is clear that

$$
\left.\begin{array}{r}
(\lambda-x) H_{\frac{2}{3}}^{(2)}\left(\frac{2}{3} \lambda^{\frac{1}{2}}(\lambda-x)^{\frac{3}{2}}\right) \\
(\lambda-x)^{\frac{1}{2}} H_{-\frac{1}{3}}^{(2)}\left(\frac{2}{3} \lambda^{\frac{1}{2}}(\lambda-x)^{\frac{3}{2}}\right)
\end{array}\right\} \in L_{2}\left(\mathbb{R}^{+}\right) .
$$


for any non-real $\lambda$ with $\Im \lambda>0$. Hence,

$$
\left.\begin{array}{l}
\theta_{1}(x, \lambda)+m(\lambda) \phi_{1}(x, \lambda) \\
\theta_{2}(x, \lambda)+m(\lambda) \phi_{2}(x, \lambda)
\end{array}\right\} \in L_{2}\left(\mathbb{R}^{+}\right)
$$

We have

$$
m(\lambda)=\frac{\mathrm{Ai}\left(-\lambda^{\frac{4}{3}}\right) \mathrm{Ai}^{\prime}\left(-\lambda^{\frac{4}{3}}\right)+\mathrm{Bi}\left(-\lambda^{\frac{4}{3}}\right) \mathrm{Bi}^{\prime}\left(-\lambda^{\frac{4}{3}}\right)+i W\left(\mathrm{Ai}\left(-\lambda^{\frac{4}{3}}\right), \mathrm{Bi}\left(-\lambda^{\frac{4}{3}}\right)\right)}{\lambda^{\frac{2}{3}}\left(\mathrm{Ai}^{2}\left(-\lambda^{\frac{4}{3}}\right)+\mathrm{Bi}^{2}\left(-\lambda^{\frac{4}{3}}\right)\right)} .
$$

Thus

$$
d \rho(\lambda)=\frac{1}{\pi} \Im m(\lambda) d \lambda=\frac{1}{\pi \lambda^{\frac{2}{3}}\left(\mathrm{Ai}^{2}\left(-\lambda^{\frac{4}{3}}\right)+\mathrm{Bi}^{2}\left(-\lambda^{\frac{4}{3}}\right)\right)} d \lambda .
$$

We arrive at the triple of integral transforms

$$
\begin{aligned}
F(\lambda)= & \pi \int_{0}^{\infty}\left\{\left[\operatorname{Bi}\left(-\lambda^{\frac{4}{3}}\right) \operatorname{Ai}^{\prime}\left(\lambda^{\frac{1}{3}} x-\lambda^{\frac{4}{3}}\right)-\operatorname{Ai}\left(-\lambda^{\frac{4}{3}}\right) \operatorname{Bi}^{\prime}\left(\lambda^{\frac{1}{3}} x-\lambda^{\frac{4}{3}}\right)\right] f_{1}(x)\right. \\
& \left.+\lambda^{\frac{2}{3}}\left[-\operatorname{Bi}\left(-\lambda^{\frac{4}{3}}\right) \operatorname{Ai}\left(\lambda^{\frac{1}{3}} x-\lambda^{\frac{4}{3}}\right)+\operatorname{Ai}\left(-\lambda^{\frac{4}{3}}\right) \operatorname{Bi}\left(\lambda^{\frac{1}{3}} x-\lambda^{\frac{4}{3}}\right)\right] f_{2}(x)\right\} d x
\end{aligned}
$$

and

$$
\left.\begin{array}{l}
f_{1}(x)=\int_{-\infty}^{\infty} \frac{\operatorname{Bi}\left(-\lambda^{\frac{4}{3}}\right) \operatorname{Ai}^{\prime}\left(\lambda^{\frac{1}{3}} x-\lambda^{\frac{4}{3}}\right)-\operatorname{Ai}\left(-\lambda^{\frac{4}{3}}\right) \mathrm{Bi}^{\prime}\left(\lambda^{\frac{1}{3}} x-\lambda^{\frac{4}{3}}\right)}{\lambda^{\frac{2}{3}}\left[\mathrm{Ai}^{2}\left(-\lambda^{\frac{4}{3}}\right)+\operatorname{Bi}^{2}\left(-\lambda^{\frac{4}{3}}\right)\right]} F(\lambda) d \lambda \\
f_{2}(x)=\int_{-\infty}^{\infty} \frac{-\operatorname{Bi}\left(-\lambda^{\frac{4}{3}}\right) \operatorname{Ai}\left(\lambda^{\frac{1}{3}} x-\lambda^{\frac{4}{3}}\right)+\operatorname{Ai}\left(-\lambda^{\frac{4}{3}}\right) \operatorname{Bi}\left(\lambda^{\frac{1}{3}} x-\lambda^{\frac{4}{3}}\right)}{\operatorname{Ai}^{2}\left(-\lambda^{\frac{4}{3}}\right)+\mathrm{Bi}^{2}\left(-\lambda^{\frac{4}{3}}\right)} F(\lambda) d \lambda
\end{array}\right\} .
$$

Theorem 1 holds for the transforms (23) if

$$
A=\left(\begin{array}{l}
0 \\
1
\end{array}\right) \quad \text { and } \quad L=\left(\begin{array}{cc}
0 & -\frac{d}{d x} \\
\frac{d}{d x} & x
\end{array}\right)
$$

Corollary 2. A vector-valued function $f$ is the $\underline{\Phi}$-transform (23) of a function $F \in L_{2}(\mathbb{R}, d \rho)$ with compact support if and only if $\underline{f}$ satisfies the following conditions:

1) $\underline{f}$ is infinitely differentiable on $\mathbb{R}^{+}$.

2) $\left(\begin{array}{cc}0 & -\frac{d}{d x} \\ \frac{d}{d x} & x\end{array}\right)^{n} \underline{f}(x) \in L_{2}\left(\mathbb{R}^{+}\right)$for all $n$.

3) $\lim _{x \rightarrow 0}(0,1) \cdot\left(\begin{array}{cc}0 & -\frac{d}{d x} \\ \frac{d}{d x} & x\end{array}\right)^{n} \underline{f}(x)=0$.

4) $\lim _{x \rightarrow \infty}\left(\begin{array}{cc}0 & -\frac{d}{d x} \\ \frac{d}{d x} & x\end{array}\right)^{n} \underline{f}(x)=0$.

5) $\lim _{n \rightarrow \infty}\left\|\left(\begin{array}{cc}0 & -\frac{d}{d x} \\ \frac{d}{d x} & x\end{array}\right)^{n} \underline{f}(x)\right\|_{L_{2}\left(\mathbb{R}^{+}\right)}^{\frac{1}{n}}<\infty$. 
Example 3 (Second Airy transform). Consider the same system (18), but with $\alpha=\frac{\pi}{2}$. The solution $\underline{\Phi}(x, \lambda)$ satisfying the initial condition

$$
\left.\begin{array}{l}
\phi_{1}(0, \lambda)=0 \\
\phi_{2}(0, \lambda)=1
\end{array}\right\}
$$

is

$$
\left.\begin{array}{l}
\phi_{1}(x, \lambda)=-\pi \lambda^{-\frac{2}{3}} \operatorname{Bi}^{\prime}\left(-\lambda^{\frac{4}{3}}\right) \operatorname{Ai}^{\prime}\left(\lambda^{\frac{1}{3}} x-\lambda^{\frac{4}{3}}\right)+\pi \lambda^{-\frac{2}{3}} \operatorname{Ai}^{\prime}\left(-\lambda^{\frac{4}{3}}\right) \operatorname{Bi}^{\prime}\left(\lambda^{\frac{1}{3}} x-\lambda^{\frac{4}{3}}\right) \\
\phi_{2}(x, \lambda)=\pi \operatorname{Bi}^{\prime}\left(-\lambda^{\frac{4}{3}}\right) \operatorname{Ai}\left(\lambda^{\frac{1}{3}} x-\lambda^{\frac{4}{3}}\right)-\pi \operatorname{Ai}^{\prime}\left(-\lambda^{\frac{4}{3}}\right) \operatorname{Bi}\left(\lambda^{\frac{1}{3}} x-\lambda^{\frac{4}{3}}\right)
\end{array}\right\}
$$

Similarly, the solution $\underline{\Theta}(x, \lambda)$ satisfying the initial condition

$$
\left.\begin{array}{l}
\theta_{1}(0, \lambda)=1 \\
\theta_{2}(0, \lambda)=0
\end{array}\right\}
$$

is

$$
\left.\begin{array}{l}
\theta_{1}(x, \lambda)=-\pi \operatorname{Bi}\left(-\lambda^{\frac{4}{3}}\right) \operatorname{Ai}^{\prime}\left(\lambda^{\frac{1}{3}} x-\lambda^{\frac{4}{3}}\right)+\pi \operatorname{Ai}\left(-\lambda^{\frac{4}{3}}\right) \operatorname{Bi}^{\prime}\left(\lambda^{\frac{1}{3}} x-\lambda^{\frac{4}{3}}\right) \\
\theta_{2}(x, \lambda)=\pi \lambda^{\frac{2}{3}} \operatorname{Bi}\left(-\lambda^{\frac{4}{3}}\right) \operatorname{Ai}\left(\lambda^{\frac{1}{3}} x-\lambda^{\frac{4}{3}}\right)-\pi \lambda^{\frac{2}{3}} \operatorname{Ai}\left(-\lambda^{\frac{4}{3}}\right) \operatorname{Bi}\left(\lambda^{\frac{1}{3}} x-\lambda^{\frac{4}{3}}\right)
\end{array}\right\} .
$$

We have

$$
\left.\begin{array}{rl}
\theta_{1}(x, \lambda)+m(\lambda) \phi_{1}(x, \lambda)= & {\left[-\operatorname{Bi}\left(-\lambda^{\frac{4}{3}}\right)-m(\lambda) \lambda^{-\frac{2}{3}} \operatorname{Bi}^{\prime}\left(-\lambda^{\frac{4}{3}}\right)\right] \pi \operatorname{Ai}^{\prime}\left(\lambda^{\frac{1}{3}} x-\lambda^{\frac{4}{3}}\right)} \\
& +\left[\operatorname{Ai}\left(-\lambda^{\frac{4}{3}}\right)+m(\lambda) \lambda^{-\frac{2}{3}} \operatorname{Ai}^{\prime}\left(-\lambda^{\frac{4}{3}}\right)\right] \pi \operatorname{Bi}^{\prime}\left(\lambda^{\frac{1}{3}} x-\lambda^{\frac{4}{3}}\right) \\
\theta_{2}(x, \lambda)+m(\lambda) \phi_{2}(x, \lambda)= & {\left[\lambda^{\frac{2}{3}} \operatorname{Bi}\left(-\lambda^{\frac{4}{3}}\right)+m(\lambda) \operatorname{Bi}^{\prime}\left(-\lambda^{\frac{4}{3}}\right)\right] \pi \operatorname{Ai}\left(\lambda^{-\frac{1}{3}} x-\lambda^{\frac{4}{3}}\right)} \\
& +\left[-\lambda^{\frac{2}{3}} \operatorname{Ai}\left(-\lambda^{\frac{4}{3}}\right)-m(\lambda) \operatorname{Ai}^{\prime}\left(-\lambda^{\frac{4}{3}}\right)\right] \pi \operatorname{Bi}\left(\lambda^{\frac{1}{3}} x-\lambda^{\frac{4}{3}}\right)
\end{array}\right\}
$$

If we take

$$
m(\lambda)=-\frac{\lambda^{\frac{2}{3}}\left(\operatorname{Ai}\left(-\lambda^{\frac{4}{3}}\right)+i \operatorname{Bi}\left(-\lambda^{\frac{4}{3}}\right)\right)}{\operatorname{Ai}^{\prime}\left(-\lambda^{\frac{4}{3}}\right)+i \operatorname{Bi}^{\prime}\left(-\lambda^{\frac{4}{3}}\right)},
$$

then the relations between the Airy and the Hankel functions (21) yield

$$
\left.\begin{array}{l}
\theta_{1}(x, \lambda)+m(\lambda) \phi_{1}(x, \lambda)=\frac{(\lambda-x) H_{\frac{2}{3}}^{(2)}\left(\frac{2}{3} \lambda^{\frac{1}{2}}(\lambda-x)^{\frac{3}{2}}\right)}{\lambda H_{\frac{2}{3}}^{(2)}\left(\frac{2}{3} \lambda^{2}\right)} \\
\theta_{2}(x, \lambda)+m(\lambda) \phi_{2}(x, \lambda)=-\frac{(\lambda-x)^{\frac{1}{2}} H_{-\frac{1}{3}}^{(2)}\left(\frac{2}{3} \lambda^{\frac{1}{2}}(\lambda-x)^{\frac{3}{2}}\right)}{\lambda^{\frac{1}{2}} H_{\frac{2}{3}}^{(2)}\left(\frac{2}{3} \lambda^{2}\right)}
\end{array}\right\} .
$$

From (22) it is clear that

$$
\left.\begin{array}{l}
\theta_{1}(x, \lambda)+m(\lambda) \phi_{1}(x, \lambda) \\
\theta_{2}(x, \lambda)+m(\lambda) \phi_{2}(x, \lambda)
\end{array}\right\} \in L_{2}\left(\mathbb{R}^{+}\right)
$$


We have

$$
m(\lambda)=-\lambda^{\frac{2}{3}} \frac{\operatorname{Ai}\left(-\lambda^{\frac{4}{3}}\right) \mathrm{Ai}^{\prime}\left(-\lambda^{\frac{4}{3}}\right)+\operatorname{Bi}\left(-\lambda^{\frac{4}{3}}\right) \mathrm{Bi}^{\prime}\left(-\lambda^{\frac{4}{3}}\right)-i W\left(\operatorname{Ai}\left(-\lambda^{\frac{4}{3}}\right), \operatorname{Bi}\left(-\lambda^{\frac{4}{3}}\right)\right)}{\mathrm{Ai}^{\prime 2}\left(-\lambda^{\frac{4}{3}}\right)+\mathrm{Bi}^{2}\left(-\lambda^{\frac{4}{3}}\right)} .
$$

Thus

$$
d \rho(\lambda)=\frac{1}{\pi} \Im m(\lambda) d \lambda=\frac{\lambda^{\frac{2}{3}}}{\pi\left(\mathrm{Ai}^{\prime 2}\left(-\lambda^{\frac{4}{3}}\right)+\mathrm{Bi}^{\prime 2}\left(-\lambda^{\frac{4}{3}}\right)\right)} d \lambda .
$$

We arrive at the triple of integral transforms

$$
\begin{aligned}
F(\lambda)= & \pi \int_{0}^{\infty}\left\{\lambda^{-\frac{2}{3}}\left[-\operatorname{Bi}^{\prime}\left(-\lambda^{\frac{4}{3}}\right) \operatorname{Ai}^{\prime}\left(\lambda^{\frac{1}{3}} x-\lambda^{\frac{4}{3}}\right)+\operatorname{Ai}^{\prime}\left(-\lambda^{\frac{4}{3}}\right) \operatorname{Bi}^{\prime}\left(\lambda^{\frac{1}{3}} x-\lambda^{\frac{4}{3}}\right)\right] f_{1}(x)\right. \\
& \left.+\left[\operatorname{Bi}^{\prime}\left(-\lambda^{\frac{4}{3}}\right) \operatorname{Ai}\left(\lambda^{\frac{1}{3}} x-\lambda^{\frac{4}{3}}\right)-\operatorname{Ai}^{\prime}\left(-\lambda^{\frac{4}{3}}\right) \operatorname{Bi}\left(\lambda^{\frac{1}{3}} x-\lambda^{\frac{4}{3}}\right)\right] f_{2}(x)\right\} d x
\end{aligned}
$$

and

$$
\left.\begin{array}{l}
f_{1}(x)=\int_{-\infty}^{\infty} \frac{-\mathrm{Bi}^{\prime}\left(-\lambda^{\frac{4}{3}}\right) \mathrm{Ai}^{\prime}\left(\lambda^{\frac{1}{3}} x-\lambda^{\frac{4}{3}}\right)+\mathrm{Ai}^{\prime}\left(-\lambda^{\frac{4}{3}}\right) \mathrm{Bi}^{\prime}\left(\lambda^{\frac{1}{3}} x-\lambda^{\frac{4}{3}}\right)}{\mathrm{Ai}^{\prime 2}\left(-\lambda^{\frac{4}{3}}\right)+\mathrm{Bi}^{\prime 2}\left(-\lambda^{\frac{4}{3}}\right)} F(\lambda) d \lambda \\
f_{2}(x)=\int_{-\infty}^{\infty} \frac{\mathrm{Bi}^{\prime}\left(-\lambda^{\frac{4}{3}}\right) \operatorname{Ai}\left(\lambda^{\frac{1}{3}} x-\lambda^{\frac{4}{3}}\right)-\mathrm{Ai}^{\prime}\left(-\lambda^{\frac{4}{3}}\right) \operatorname{Bi}\left(\lambda^{\frac{1}{3}} x-\lambda^{\frac{4}{3}}\right)}{\operatorname{Ai}^{\prime 2}\left(-\lambda^{\frac{4}{3}}\right)+\mathrm{Bi}^{\prime 2}\left(-\lambda^{\frac{4}{3}}\right)} F(\lambda) \lambda^{\frac{2}{3}} d \lambda
\end{array}\right\} .
$$

Theorem 1 holds for transforms (24) if

$$
A=\left(\begin{array}{l}
1 \\
0
\end{array}\right) \quad \text { and } \quad L=\left(\begin{array}{cc}
0 & -\frac{d}{d x} \\
\frac{d}{d x} & x
\end{array}\right)
$$

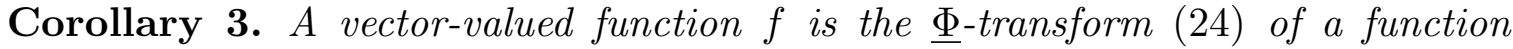
$F \in L_{2}(\mathbb{R}, d \rho)$ with compact support if and only if $\underline{f}$ satisfies the following conditions:

1) $\underline{f}$ is infinitely differentiable on $\mathbb{R}^{+}$.

2) $\left(\begin{array}{cc}0 & -\frac{d}{d x} \\ \frac{d}{d x} & x\end{array}\right)^{n} \underline{f}(x) \in L_{2}\left(\mathbb{R}^{+}\right)$for all $n$.

3) $\lim _{x \rightarrow 0}(1,0) \cdot\left(\begin{array}{cc}0 & -\frac{d}{d x} \\ \frac{d}{d x} & x\end{array}\right)^{n} \underline{f}(x)=0$.

4) $\lim _{x \rightarrow \infty}\left(\begin{array}{cc}0 & -\frac{d}{d x} \\ \frac{d}{d x} & x\end{array}\right)^{n} \underline{f}(x)=0$.

5) $\lim _{n \rightarrow \infty}\left\|\left(\begin{array}{cc}0 & -\frac{d}{d x} \\ \frac{d}{d x} & x\end{array}\right)^{n} \underline{f}(x)\right\|_{L_{2}\left(\mathbb{R}^{+}\right)}^{\frac{1}{n}}<\infty$.

Acknowledgement. This work was accomplished while Dr. Zayed was visiting the Department of Mathematics and Computer Science, Faculty of Science, Kuwait University, and he would like to take this opportunity to thank the department for its hospitality. The work of the second named author was supported by the Kuwait University Research Administration under the research grant SM 187. 


\section{References}

[1] Abramowitz, M. and I. A. Stegun: Handbook of Mathematical Functions, with Formulas, Graphs, and Mathematical Tables. New York: Dover Publication 1972.

[2] Bang, H. H.: A property of infinitely differentiable functions. Proc. Amer. Math. Soc. 108 (1990), $73-76$.

[3] Hinton, D. and J. Shaw: Hamiltonian systems of limit point or limit circle type with both end points singular. J. Diff. Eqs. 50 (1983), $444-464$.

[4] Levitan, B. M. and I. S. Sargsjan: Sturm-Liouville and Dirac Operators. Dordrecht: Kluwer Acad. Publ. 1991.

[5] Paley, R. E. A. C. and N. Wiener: Fourier Transforms in the Complex Domain. Providence (R.I., USA): Amer. Math. Soc. 1934.

[6] Titchmarsh, E. C.: Introduction to the Theory of Fourier Integrals. New York: Chelsea Publ. Comp. 1986.

[7] Titchmarsh, E. C.: Eigenfunction Expansions Associated with Second-Order Differential Equations, Part 1. Oxford: Clarendon Press 1962.

[8] Tuan, V. K.: Supports of functions and integral transforms. In: Proc. Int. Workshop on Recent Adv. Appl. Math. (RAAM' 96) held in Kuwait May 4 - 7, 1996. Kuwait: University 1996, pp. $507-521$.

[9] Tuan, V. K.: On the range of the Y-transform. Bull Austral. Math. Soc. 54 (1996), 329 -345 .

[10] Tuan, V. K.: On the range of the Hankel and extended Hankel transforms. J. Math. Anal. Appl. 209 (1997), $460-478$.

[11] Tuan, V. K.: New type Paley-Wiener theorems for the modified multidimensional Mellin transform. J. Fourier Anal. Appl. 4 (1998), 317 - 328.

[12] Tuan, V. K.: Airy integral transform and the Paley-Wiener theorem. In: Proc. 2nd Int. Workshop on Transform Methods and Special Functions, Varna (Bulgaria) 23 - 30 August 1996 (eds.: D. Rusev D. et al.). Sofia: IMI-BAS 1998, pp. 523 - 531.

[13] Tuan, V. K. and A. I. Zayed: Paley-Wiener-type theorems for a class of integral transforms (submitted).

Received 09.08.1999 